\title{
Micro RNAs- A Review
}

Sathya Selvarajan ${ }^{1}$, Jaya Vijayaraghavan², Zachariah Bobby³, Jothimalar Ramalingam ${ }^{4}$

${ }^{1}$ Assistant Professor, Department of Biochemistry, Saveetha Medical College and Hospital, SIMATS, Thandalam, Chennai, Tamilnadu, India. ${ }^{2}$ Professor, Department of Obstetrics and Gynaecology, SRMC\&RI, Porur, Chennai, Tamilnadu, India. ${ }^{3}$ Professor, Department of Biochemistry, JIPMER, Puducherry, India. ${ }^{4}$ Professor, Department of Biochemistry, SRMC\&RI, Porur, Chennai, Tamilnadu, India.

\section{ABSTRACT}

\section{BACKGROUND}

MicroRNAs (miRs) belong to a category of small non-coding RNAs that perform important functions as gene regulators. These miniscule, roughly $\sim 20$ nucleotide RNAs have been phylogenetically well preserved and have widespread roles in transcription and translation. Since discovery in 1993, numerous miRs in various species have been documented and a common nomenclature has been adopted. Biogenetic pathways of these unique small RNAs are divided into canonical and noncanonical pathways, based on their dependence or independence on Drosha/DGCR8 and Dicer for processing. The common method of gene silencing is via mRNA cleavage or translational repression. In certain scenarios, they have also been found to activate protein synthesis. The dynamic interactions of miRs with their target DNA depends on numerous factors like their affinity, location and abundance. The turnovers of miRs are dictated by numerous modifications like uridylation and adenylation. Whenever new miRs are detected, their expression, conservation and experimental quantification are used to find novel gene pathways and their target mRNA. MiRs have been released into circulation, either in vesicles or bound to proteins. Extracellular presence of miRs may be a consequence of cell death or injury; but more regulated processes like endocytosis and pinocytosis also seem to have some role. The variation in expression and type of circulating miRs has been studied as markers of disease manifestations.

\section{KEY WORDS}

MicroRNA, Canonical Pathway, Extracellular miR, Circulating miR, Post Transcriptional Gene Regulation, Gene Silencing, RT-PCR, Gene Expression Regulation, Biomarker
Corresponding Author:

Dr. Jothimalar Ramalingam,

Professor,

Department of Biochemistry,

SRMC\&RI, Porur, Chennai-600116,

Tamilnadu, India.

E-mail:drjothimalar@gmail.com

DOI: 10.14260/jemds/2019/634

Financial or Other Competing Interests: None.

How to Cite This Article:

Selvarajan S, Vijayaraghavan J, Bobby Z, et al. Micro RNAs- a review. J. Evolution Med. Dent. Sci. 2019;8(38):2918-2923, DOI: 10.14260/jemds/2019/634

Submission 24-07-2019,

Peer Review 05-09-2019,

Acceptance 12-09-2019,

Published 23-09-2019. 


\section{BACKGROUND}

MicroRNAs (miRs) are small non-coding RNA molecules about 19-25 nucleotides in length that are seen in plants, animals and a few viruses. They act as guiding molecules in post transcriptional mechanisms of regulating gene expression.(1) Their action is exerted through base pairing of complimentary sequences in mRNA. In consequence, silencing of mRNA occurs via one or more of these: 1) mRNA cleavage, 2) Poly(A) tail shortening destabilizing the mRNA and 3) less effective translation process due to repression (). MiRs look similar in appearance to the small interfering RNA (siRNA) in the RNA interference pathway. However, miRs are generated from the short hairpin shaped transcripts while siRNA originate from the double stranded RNA's longer portions.(2) Since discovery, a minimum of $200 \mathrm{miRs}$ have been identified in each species accounting for approximately $1 \%$ of the entire genome.(2) In the humans alone, over $1900 \mathrm{miRs}$ are said to have been encoded in the genome, (3) but recent studies quote only about 600.(4) They are seen in abundance in numerous mammalian cells(5) and appear to target around sixty percent of genes.(6) Evolutionary conservation of miRs implies that they may have vital biological roles.(4) Mouse knockout studies show pertinent functions of around 90 families of such conserved miRs with a common ancestry in fish and mammals.(7) MiRs are significant for development and partake in various biological processes.(8) Abnormal variation in miRs is associated with diseases like breast cancer, multiple sclerosis, diabetes and Alzheimer's disease. $(9,10)$ In addition, miRs are secreted extracellularly, wherein they act as signalling molecules mediating communication between cells.(11)

\section{History}

Although the first miR discovery dates back to the early $1990 s,(12)$ they weren't accepted as a group of regulators of biological action until the 2000s.(13) Different miRs were found to be expressed in various cells and tissues $(5,14)$ exerting numerous roles ${ }^{(15,16,17)}$ and implicated in diseases like chronic lymphocytic leukemia as well. Currently scientists are exploring therapeutic interventions based on miRs.(18) Discovery of lin-4, the first miR in Caenorhabditis elegans by Lee, Feinbaum and Ambros, over 25 years ago in 1993 was the beginning. At that time, it was thought of as a nematode idiosyncrasy. Ruvkun et al were the team who identified its mode of action in the development of C. elegans larvae by repression of the lin-14 gene.(12,19) Again in 2000, another small RNA let-7 was identified to repress lin-41.(20) All these evidence pointed to the fact that such small temporal RNAs may regulate growth and development in numerous species. After a year, these two miRs were found to belong to a larger sect of such similar small RNAs possessing various regulatory roles, were identified not only in C. elegans but also in Drosophila and human cell lines.(13) This was when the term miR was coined.

\section{Nomenclature}

A standardized nomenclature system was adopted in 2003 to assign names to experimentally confirmed new miRs prior to publishing.(21) The term miR followed by a hyphen and an indicated number was the preferred way of ordaining. The pre and pri miRs were indicated by 'mir'; 'miR' denoted the mature micro RNA and 'MIR' referred to the genes that encoded those
miR.(22) When miRs had almost similar sequences except for a few nucleotides they were denoted with an additional alphabet in lower case e.g., miR-146a and miR-146b. Identical miRs at different sites were annotated with an additional hyphenated number at the end, e.g. miR 121-1 and 121-1. A three lettered prefix was used to indicate the species of origin, e.g. hsa-miR-123 for Homo sapiens, $v$ for viral and $d$ for Drosophila, commonly called fruit fly. Two miRs from opposite arms of the same pre-miR in nearly similar amounts were symbolized with a $-3 p$ or $-5 p$ at the end. But mostly the miR from one arm of the hairpin was more plentiful than the other. The miR in lesser quantity was denoted by an asterisk after the name.

\section{miR Genes}

Mostly miR genes are situated in the intergenic region more than $1 \mathrm{~kb}$ away from the indicated gene; however a few are placed in the introns of genes in the sense or anti-sense orientation and regulated along with their host genes.(23) Therefore it was concluded that most of the miR genes may be transcribed as independent units. Since nearly half the miR were found in close proximity to their peers, it raised a possibility of a single polycistronic transcription unit. MiR genes were found to have their own promoters.

\section{Biogenesis}

The biogenesis of $\mathrm{miR}$ is broadly divided into canonical (usually dominant pathway) and non-canonical pathways (independent of Drosha/DGCR8 and Dicer).

\section{Canonical Pathway of Biogenesis}

Transcription

miRNA genes are transcribed normally by RNA polymerase II (Pol II) but other RNA polymerases do transcribe a minority of them.(24) RNA Polymerase III transcribes certain miR with upstream Alu sequences. Pol II adheres to a promoter very close to the DNA sequence that encodes the pre-miR. This premiR undergoes post-transcriptional modifications like capping at the $5^{\prime}$ end, poly (A) tail(24) and splicing The initial product is the primary miR or pri-miR which is several $\mathrm{kb}$ long containing one or more stem loops structures. These stem loops are roughly 80 nucleotides long with accompanying sequences required for processing.

Nuclear processing The pri-miR is recognized within the nucleus by a protein DiGeorge Syndrome Critical Region 8 (DGCR8 or termed "Pasha" among invertebrates), named after its relation to DiGeorge Syndrome. DGCR8 is a $\sim 120 \mathrm{kDa}$ protein which has two dsRBDs and a WW domain interacting specifically with sequences rich in proline. The exact function of DGCR8 is unclear but it identifies substrate dsRNA assemblies of the hairpins in a pri-miR.(25) DGCR8 associates with Drosha, a $\sim 160 \mathrm{kDa}$ protein, highly conserved in animals. Drosha has two tandem RNase III domains (RIIIDs), a double stranded RNA binding domain (dsRBD) which are both vital for catalytic activity; and a central region next to the RIIIDs also necessary for the processing of pri-miR. This enzyme cleaves the RNA forming the Microprocessor complex of $\sim 650$ $\mathrm{kDa}$ in humans. Within this complex, Drosha interacts with DGCR8 which directs the RNase III domain within Drosha to release hairpins via cleaving RNA roughly 11 nucleotides away from the base of the hairpin. The resultant is left with a 2 nucleotide projection at 3' end; 3' hydroxyl and 5' phosphate 
groups. It's called the pre-miR (Precursor-miR). Around 16\% of pre-miR undergo RNA editing, which stops nuclear processing and alters cytosolic processing and target specificity. For example, adenosine deaminases acting on RNA (ADARs) catalyse the transition of adenosine to inosine.(26)

\section{Export to Cytoplasm}

Pre-miRs are exported from the nucleus into the cytoplasm in a crucial step by the Shuttler Exportin-5. This is an energydependent process using GTP which is bound to the Ran protein.(27) This protein of the karyopherin family, distinguishes a two-nucleotide overhang at the 3 ' end of the pre-miR.

\section{Cytoplasmic Processing}

Once in the cytoplasm, the pre-miR is cleaved by another RNase III enzyme called Dicer. ${ }^{(28)}$ Dicer has 6 known domains: two RIIIDs and a dsRBD along with a long $\mathrm{N}$ terminal segment which has a dead-box RNA helicase domain, DUF283 domain and a PAZ domain. The PAZ domain binds to the 3' end of small RNAs while the role of the other domains are unclear.(28) This enzyme snips the $5^{\prime}$ and $3^{\prime}$ arms producing an inconsistent miRNA: miRNA* duplex around 22 nucleotides in length. The flawed pairing, length of the hairpin loop and the loop size all affect the efficiency of the Dicer activity. Dicer action is combined with duplex unwinding and one strand selected based on thermodynamic instability, position of stem loop and weaker base pairing on the 5'end is loaded into the RNAinduced silencing complex (RISC) where the miR and its target mRNA interact. The other passenger strand denoted with the asterisk is degraded usually but in rare cases, both strands become viable and functional miR. RISC complex contains Dicer and related proteins. RISC is otherwise called microRNA ribonucleoprotein complex (miRNP); a RISC with the miR is often termed miRISC. miRISC and target mRNA have been found in various subcellular compartments like rough endoplasmic reticulum (rER),(29) processing (P)-bodies, $(30)$ stress granules (SG),(31) the trans-Golgi network (TGN), early/late endosomes,(32) multivesicular bodies (MVB),(33) lysosomes,(33) mitochondria,(34) and the nucleus.(35) The constituents that modulate miR post-transcriptional gene regulation are enhanced at places where miRISC: mRNA complexes are present. Argonaute (Ago) protein family plays a vital role in RISC activity. They are 8 in number in humans divided into the AGO and PIWI families.(36) They are required for miR-induced silencing. They have 2 conserved binding sites - a PAZ domain and a PIWI domain. The former bind the 3 ' end of mature miR; while the latter looks structurally similar to ribonuclease- $\mathrm{H}$ and binds with the 5'end. Ago proteins direct the miR for target mRNA binding either directly or by recruiting more proteins to cause repression.(36)

\section{Non-Canonical Pathways of Biogenesis}

Broadly, the non-canonical pathways may be divided into Drosha/DGCR8-independent and Dicer-independent pathways. Pre-miRs resulting from Drosha/DGCR8independent pathway look similar to Dicer substrates. An example is miRs generated at the time of splicing from introns of mRNA, without forming the Microprocessor complex called Mirtrons.(37) Formerly assumed to occur merely in C. elegans and fruit fly, mirtrons have been identified in mammals too.(37) Another example is 7-methyl guanosine $\left(\mathrm{m}^{7} \mathrm{G}\right)$ capping of premiR. Such capped miRs are straightaway exported into the cytoplasm through exportin 1 without undergoing cleavage by Drosha.(38) MiRs independent of Dicer are processed via Drosha from transcripts of short hairpin RNA called shRNA .(39) These pre-miRs need Ago2 in order to mature in the cytoplasm as their length is inadequate to be Dicer substrates.(39) Their maturation is complete by trimming of the $5 p$ strand in the $3^{\prime}$ $5^{\prime}$ direction.

\section{Action of miR on mRNA and GENES}

Almost always, miRs act on the 3' untranslated region (UTR) of their targeted mRNAs to suppress expression. But miR interaction with other sites like coding sequences, 5' UTR and promoter sequences have been documented too. In some instances, miRs are involved in activation of gene expression too. In recent times, miRs have been found to shuttle between the various subcellular compartments to monitor rates of transcription and translation.(40) A combination of different types of mRNA regulation by miRs happen in animals. One miR can have numerous mRNA targets and vice versa, one target may be regulated by more than one miR. Work by Bartel and Burge indicated that nearly $1 / 3$ rd of the entire human genome is targeted by miRs. ${ }^{(41)}$ Gene silencing can take place via either of two routes - degradation of mRNA or by preventing the occurrence of mRNA translation depending on whether there is a complete complimentary of the miRNA and the mRNA target sequences or not. The concepts of coherent feedforward loop, double negative feedback loop and positive feedback/feed-forward loop explains the association of miR and its target. Gene expression due to a random event in the genetic machinery is buffered by some miRs by a negative feed loop or incoherent feed-forward loop which uncouples protein synthesis from transcription. Activation of gene expression via dsRNA is called small RNA-induced gene activation (RNAa). This was seen in endogenous miRs, and also in cells invitro using synthesized dsRNA called small activating RNA (saRNA) where they targeted promoter sequences to potentially activate transcription of related genes.(42) Competing endogenous RNAs (ceRNAs) are miRs that interact with miR response elements on genes and pseudogenes, thereby creating an alternative method for regulating gene expression among paralogous genes.

\section{Turnover}

The stability or decay of miRs is dependent on numerous modifications which may cause them. In C. elegans, the $5^{\prime}$ to $3^{\prime}$ exoribonuclease Rat1p or XRN2 was found to mediate the decay of mature miRs. Small degrading nucleases (SDNs) acting in the opposite direction have been reported but their functions haven't been studied in detail.(43) Uridylation degrades miRs but this is prevented by 2'-0-conjugated methyl groups. But in certain cases uridylation has been found to prevent degradation too, the reason for which is not understood. Adenylation in the liver enriching mammalian miR-122 (pertinent in hepatitis C) was found to stabilize the molecule.(43)

\section{Functions}

Morozova et al created a unified mathematical model from previous literature and deduced the mode of action of miRs. 
They can cause mRNA destabilization and cleavage; inhibition of transcription by chromatin reorganization; inhibiting initiation of $40 \mathrm{~S}$ cap, joining of $60 \mathrm{~S}$ ribosomal unit and elongation. It also influences translation by premature termination of ribosome drop-off and nascent protein degradation. Post translationally the proteins can be sequestered in $\mathrm{P}$ bodies. DNA methylation and histone modification by miRs also affect the target genes. IsomiRs are miRs expressing RNA editing which cause modification of RNA sequences at specific sites giving products dissimilar to that intended by the original DNA. This leads to a vast implication of the miR action outside their respective genomes themselves.

\section{Phylogenetic Conservation}

In both plants and animals, miRs have been phylogenetically well conserved, so they are considered a fundamentally necessary component of gene regulation.(44) Though essentially similar in pathways in both plants and animals, evolutionary transformation in their basic mode of action has differentiated their roles. And due to their low rate of evolution, miRs may have led to innovations in morphology and also allowed finer changes in gene expression to take place ultimately resulting in the biogenesis of intricate organs and may have helped in the generation of complex organisms. ${ }^{(44)}$ Yet again, this is evidenced by the increase in miRs during spurts of morphological development.(45) Novel miRs are produced within the living cell by arbitrary hairpin development in the intergenic regions of DNA or by duplication and modification of prevailing miRs. A fold back hairpin assembly can be fabricated by reverse replicating a protein coding sequence. Older miRs undergo a rather slower rate of substitution (Sometimes one change in a hundred million years) conveying that miRs after gain-of-function may undergo a purifying selection process. Individual areas within one developmental miR gene may have varying developmental rates. MiRs are seldom lost from an organism's genome but newer non-functional miRs are frequently lost. While bacteria lack true miRs, nearly 5000 vagaries of miRs have been reported up until March 2010 across species.(46)

\section{Techniques to Study miRs}

Practically speaking, whenever a small RNA is identified by cDNA cloning, it should meet certain criteria to qualify as a miR. First, it's important to confirm its expression by hybridizing with size-fractionated RNA, most commonly done with Northern blotting as it shows both the mature miR and the precursor form with approximately a 70 nucleotide band. The other methods available for the same are Reverse Transcription Polymerized Chain Reaction (RT-PCR), microarray, RNase protection assay and primer extension analysis. Secondly, this small sequence of RNA must exist in one arm of the hairpin precursor. This precursor length is about 60-80 nucleotides in animals but variable in plants, and has no internal loops or bulges. Thirdly this sequence should be conserved phylogenetically and should be seen in the precursor form rather than in the mature form. A fourth criterion not commonly used due to difficulties in technicality of performing, is the accumulation of the precursor following depletion/reduced function of the RNase-III type enzyme Dicer. Singularly none of these criteria can be used to label a candidate RNA as a novel miR. Commonly the expression and structure or expression and conservation are taken to be adequate. If expression is extremely low to detect, then the phylogenetic conservation along with cDNA cloning is deemed adequate. If any other method is used, then expression should be established with conservation and structure of precursor. If detection is impossible and only anticipated, then the fourth criterion of precursor accumulation should be demonstrated. MiR orthologues are similar homologues in different species which can be accepted without validating experimentally, only if the conservation criterion is satisfied. Small RNAs which do not qualify based on these criteria are termed siRNAs or other provisionary classes. The list of miRs is expanding rapidly due to expansive cloning and computational software based prediction methods. Profiling and discovery of new miRs is done by high throughput sequencing methods; 'in situ detection' and activity measurement by experimental inhibition with a locked nucleic acid (LNA) oligo, Morpholingo oligo,(47) or a 2'-0-methyl RNA oligo.(47) Due to their short length and omnipresent RNases, miRs degrade more easily than mRNAs. It is obligatory to use RNase free instruments and cool the samples on ice. MiRs are generally quantified by a twostep RT-PCR and subsequently by quantitative PCR. Adaptations in this technique can bring relative or absolute quantification. Microarray hybridization on slides or chips with probes to in numerous miR targets can also be used. In order to identify target mRNA, complementary antagomirs (Which silence a particular miR) and steric blocking oligos (That block maturation of miRs and action on target mRNA) can be used.(48) Several databases are available online to track and predict mRNA targets for miRs based on their base sequence. ${ }^{(49)}$ Generally this is undertaken once novel miRs are detected but research is underway to incorporate mRNA and miR expression information ().

\section{Circulating miRs}

Some extracellular miRs are derivatives of cellular activities like cell injury or death, but there is a lot of evidence pointing to a regulated process of miR release. Studies show that exosomal miRs are facilitated by a ceramide-dependent pathway and such miRs have a growth regulatory effect on targeted cells. The mechanisms of circulating miRs are being researched nowadays. Studies point out that vesicle-mediated extracellular miRs enter cells by phagocytosis, micropinocytosis, endocytosis or by directly fusing with plasma membranes; and there are vesicle-free secreted miRs whose uptake is by distinctive receptors on surface of cells. Extracellular miRs have been implicated as autocrine, paracrine and/or endocrine regulators of target cell actions, thereby indicating that miRs possess hormone-like actions. Extra cellularly, miRs have been identified in various biological fluids like plasma, tears, serum, urine, saliva, seminal fluid, breast milk, bronchial lavage, cerebrospinal fluid, peritoneal fluid and ovarian follicular fluid. In circulation in biological fluids, miRs are found either in vesicles like the exosomes, micro vesicles and apoptotic bodies or bound to proteins like high density lipoprotein (HDL), nucleophosmin 1 (NPM1) and Ago 2. Due to their binding to proteins or presence in vesicles, extracellular circulating miRs are highly stable, resisting numerous freeze-thaw cycles, boiling, changes in $\mathrm{pH}$ and do not degrade in room temperature for nearly 4 days.(50) 


\section{miRs in Circulation as Diseases Markers}

A dysregulation of miRs and corresponding changes in their concentration in blood has been associated with disease conditions. miR2 Disease is a database which is publicly available that documents associations of various miR dysregulations with diseases in humans.(51)

\section{CONCLUSIONS}

MiRs function not only as gene regulators but also influence the entire genetic machinery from transcription to protein synthesis. The target genes of various miRs and the pathways affected and proteins secreted have been elucidated. However, our knowledge of how exactly they influence these mechanisms is unclear and a lot of the literature available is mostly speculative. Real time viewing of movement of miRs in high special and temporal resolution will shed further light into the complex dynamics of this novel small RNA. Similarly circulating miRs have been found to reflect the physiological and pathological status of tissues and suggested to be used in identification of diseases. The methods of secretion of extracellular miRs and how some of them interact with cell surface receptors have to be studied in depth to understand their usage as markers in disease conditions.

\section{REFERENCES}

[1] Ambros V. The functions of animal microRNAs. Nature 2004;431(7006):350-5.

[2] Bartel DP. MicroRNAs: genomics, biogenesis, mechanism and function. Cell 2004;116(2):281-97.

[3] Homo sapiens miRNAs in the miRBase at Manchester University. 1917 sequeneces.

[4] Fromm B, Billipp T, Peck LE, et al. An uniform system for the annotation of vertebrate microrna genes and the evolution of the human microRNAome. Annual Review of Genetics 2015;49:213-42.

[5] Lim LP, Lau NC, Weinstein EG, et al. The microRNAs of Caenorhabditis elegans. Genes \& Development 2003;17(8):991-1008.

[6] Friedman RC, Farh KK, Burge CB, et al. Most mammalian mRNAs are conserved targets of microRNAs. Genome Research 2009;19(1):92-105

[7] Bartel DP. Metazoan MicroRNAs. Cell 2018;173(1):20-51.

[8] Fu G, Brkic J, Hayder $\mathrm{H}$, et al. MicroRNAs in human placental development and pregnancy complications. Int J Mol Sci 2013;14(3):5519-44.

[9] Tufekci KU, Oner MG, Meuwissen RL, et al. The role of microRNAs in human diseases. Methods Mol Biol 2014;1107:33-50.

[10] Paul P, Chakraborty A, Sarkar D, et al. Interplay between miRNAs and human diseases. J Cell Physiol 2018;233(3):2007-18.

[11] Huang W. MicroRNAs: biomarkers, diagnostics and therapeutics. Methods Mol Biol 2017;1617:57-67.

[12] Lee RC, Feinbaum RL, Ambros V. The C. elegans heterochronic gene lin-4 encodes small RNAs with antisense complementarity to lin-14. Cell 1993;75(5):843-54.
[13] Lee RC, Ambros V. An extensive class of small RNAs in Caenorhabditis elegans. Science 2001;294(5543):862-4.

[14] Wienholds E, Kloosterman WP, Miska E, et al. MicroRNA expression in zebrafish embryonic development. Science 2005;309(5732):310-1.

[15] Jones-Rhoades MW, Bartel DP, Bartel B. MicroRNAS and their regulatory roles in plants. Annual Review of Plant Biology 2006;57:19-53.

[16] Brennecke J, Hipfner DR, Stark A, et al. Bantam encodes a developmentally regulated microRNA that controls cell proliferation and regulates the proapoptotic gene hid in Drosophila. Cell 2003;113(1):25-36.

[17] Cuellar TL, McManus MT. MicroRNAs and endocrine biology. The Journal of Endocrinology 2005;187(3):32732.

[18] Hydbring P, Badalian-Very G. Clinical applications of microRNAs. F1000 Research 2010;2:136.

[19] Wightman B, Ha I, Ruvkun G. Post-transcriptional regulation of the heterochronic gene lin-14 by lin-4 mediates temporal pattern formation in C. elegans. Cell 1993;75(5):855-62.

[20] Reinhart BJ, Slack FJ, Basson M, et al. The 21-nucleotide let-7 RNA regulates developmental timing in Caenorhabditis elegans. Nature 2000;403(6772):901-6.

[21] Griffiths-Jones S, Grocock RJ, van Dongen S, et al. miRBase: microRNA sequences, targets and gene nomenclature. Nucleic Acids Research 2006;34(Database issue):D140-4

[22] Wright MW, Bruford EA. Naming 'junk': human nonprotein coding RNA (ncRNA) gene nomenclature. Human Genomics 2011;5(2):90-8.

[23] Kim YK, Kim VN. Processing of intronic microRNAs. The EMBO Journal 2007;26(3):775-83.

[24] Cai X, Hagedorn CH, Cullen BR. Human microRNAs are processed from capped, polyadenylated transcripts that can also function as mRNAs. RNA 2004;10(12):1957-66.

[25] Gregory RI, Yan KP, Amuthan G, et al. The Microprocessor complex mediates the genesis of microRNAs. Nature 2004;432(7014):235-40.

[26] Winter J, Jung S, Keller S, et al. Many roads to maturity: microRNA biogenesis pathways and their regulation. Nature Cell Biology 2009;11(3):228-34.

[27] Murchison EP, Hannon GJ. miRNAs on the move: miRNA biogenesis and the RNAi machinery. Current Opinion in Cell Biology 2004;16(3):223-9.

[28] Ji X. The mechanism of RNase III action: how dicer dices. Current Topics in Microbiology and Immunology 2008;320:99-116.

[29] Barman B, Bhattacharyya SN. mRNA targeting to endoplasmic reticulum precedes ago protein interaction and microRNA (miRNA)-mediated translation repression in mammalian cells. J Biol Chem 2015;290(41):24650-6.

[30] Nishi K, Takahashi T, Suzawa M, et al. Control of the localization and function of a miRNA silencing component TNRC6A by Argonaute protein. Nucleic Acids Res 2015;43(20):9856-73.

[31] Detzer A, Engel C, Wunsche W, et al. Cell stress is related to re-localization of Argonaute 2 and to decreased RNA interference in human cells. Nucleic Acids Res 2011;39(7):2727-41. 
[32] Bose M, Barman B, Goswami A, et al. Spatiotemporal uncoupling of MicroRNA-mediated translational repression and target RNA degradation controls microRNP recycling in mammalian cells. Mol Cell Biol 2017;37(4):e00464-16.

[33] Gibbings DJ, Ciaudo C, Erhardt M, et al. Multivesicular bodies associate with components of miRNA effector complexes and modulate miRNA activity. Nat Cell Biol 2009;11(9):1143-9.

[34] Zhang X, Zuo X, Yang B, et al. MicroRNA directly enhances mitochondrial translation during muscle differentiation. Cell 2014;158(3):607-19.

[35] Miao L, Yao H, Li C, et al. A dual inhibition: microRNA-552 suppresses both transcription and translation of cytochrome P450 2E1. Biochim Biophys Acta 2016;1859(4):650-62.

[36] Pratt AJ, MacRae IJ. The RNA-induced silencing complex: a versatile gene-silencing machine. The Journal of Biological Chemistry 2009;284(27):17897-901.

[37] Berezikov E, Chung WJ, Willis J, et al. Mammalian mirtron genes. Molecular Cell 2007;28(2):328-36.

[38] Xie M, Li M, Vilborg A, et al. Mammalian 5'capped microRNA precursors that generate a single microRNA. Cell 2013;155(7):1568-80.

[39] Yang JS, Maurin T, Robine N, et al. Conserved vertebrate mir-451 provides a platform for Dicer-independent, Ago2-mediated microRNA biogenesis. Proc Natl Acad Sci USA 2010;107(34):15163-8.

[40] Makarova JA, Shkurnikov MU, Wicklein D, et al. Intracellular and extracellular microRNA: an update on localization and biological role. Prog Histochem Cytochem 2016;51(3-4):33-49.

[41] Lewis BP, Burge CB, Bartel DP. Conserved seed pairing, often flanked by adenosines, indicates that thousands of human genes are microRNA targets. Cell 2005;120(1):1520.

[42] Li LC. Small RNA-mediated gene activation. In Morris KV, edr. RNA and the regulation of gene expression: a hidden layer of complexity. Horizon Scientific Press 2008. ISBN 978-1-904455-25-7.

[43] Kai ZS, Pasquinelli AE. MicroRNA assassins: factors that regulate the disappearance of miRNAs. Nature Structural \& Molecular Biology 2010;17(1):5-10.

[44] Peterson KJ, Dietrich MR, McPeek MA. MicroRNAs and metazoan macroevolution: insights into canalization, complexity and the Cambrian explosion. BioEssays 2009;31(7):736-47.

[45] Wheeler BM, Heimberg AM, Moy VN, et al. The deep evolution of metazoan microRNAs. Evolution \& Development 2009;11(1):50-68.

[46] Dimond PF. miRNAs' therapeutic potential. Genetic Engineering \& Biotechnology News 2010;30(6):1.

[47] Meister G, Landthaler M, Dorsett Y, et al. Sequencespecific inhibition of microRNA- and siRNA-induced RNA silencing. RNA 2004;10(3):544-50.

[48] Gebert LF, Rebhan MA, Crivelli SE, et al. Miravirsen (SPC3649) can inhibit the biogenesis of miR-122. Nucleic Acids Research 2014;42(1):609-21.

[49] Artmann S, Jung K, Bleckmann A, et al. Detection of simultaneous group effects in microRNA expression and related target gene sets. PLoS One 2012;7(6): e38365.

[50] Mitchell PS, Parkin RK, Kroh EM, et al. Circulating microRNAs as stable blood-based markers for cancer detection. Proc Natl Acad Sci USA 2008;105(30):10513-8.

[51] Jiang Q, Wang Y, Hao Y, et al. miR2Disease: a manually curated database for microRNA deregulation in human disease. Nucleic Acids Research 2009;37(Database issue):D98-104. 